\title{
Studying Heart Rate Variability from Ballistocardiography Acquired by Force Platform: Comparison with Conventional ECG
}

\author{
Alba Martín-Yebra ${ }^{1,2}$, Federica Landreani ${ }^{1}$, Claudia Casellato ${ }^{1}$, Esteban Pavan ${ }^{1}$, Carlo Frigo $^{1}$, \\ Pierre-François Migeotte ${ }^{3}$, Enrico G Caiani ${ }^{1}$ \\ ${ }^{1}$ Dipartimento di Elettronica, Informazione e Bioingegneria, Politecnico di Milano, Milan, Italy \\ ${ }^{2}$ Aragón Institute of Engineering Research, IIS Aragón, Universidad de Zaragoza, Zaragoza, Spain \\ ${ }^{3}$ Université libre de Bruxelles, Brussels, Belgium
}

\begin{abstract}
Ballistocardiography (BCG), measuring the heart beatinduced mass movements of the body generated by forces associated to heart contraction, has been recently reconsidered as a useful non-invasive technique to characterize cardiac performance, potentially offering a simple, efficient and affordable solution for e-health monitoring. Our aim was to extract heart rate variability indices from $B C G$ signal (B-HRV), and to evaluate their correlation with ECG-derived (E-HRV) ones, both in time and frequency domains.

Five-minute BCG signals from 18 healthy volunteers were acquired by a piezoelectric 3D-force plate simultaneously with the ECG signal, in both standing (ST) and supine (SUP) positions. BCG systolic waves (H, I, J and $K$ ) in the longitudinal (head-foot) component were detected, from which B-HRV series were computed and compared by correlation and Bland-Altman analysis against conventional RR.

Posture affected accuracy of B-HRV time and frequency parameters compared to ECG-HRV, with the best performance obtained in SUP by the JJ series. This signal could be used as an alternative measurement of HRV in novel home e-health monitoring systems.
\end{abstract}

\section{Introduction}

Cardiac function, which is regulated by the autonomic nervous system (ANS), is commonly assessed by using non-invasive techniques, being heart rate variability (HRV) the most widely-used and reliable marker of the status of both sympathetic and parasympathetic branches of the ANS [1]. The importance of daily cardiac monitoring for preventing and early-detecting cardiac-related diseases has already been addressed. However, some constraints such as the need of adhesive electrodes, the time for placement, or even the requirement of medical staff, makes the ECG practice non suitable for everyday usage, despite it yields good results in clinical practice.

In this context, the ballistocardiogram (BCG), which measures the heart beat-induced mass movements of circulating blood and the heart itself, being generated by the acceleration of blood as it is ejected and moved to the aorta and the large vessels [2], has been recently reconsidered as a useful non-invasive technique to characterize cardiac performance. This technique potentially offers a simple, efficient and affordable solution for home e-health monitoring as it can be embedded in commonly utilized objects.

Our aim in this work was to extract beat-to-beat HRV series from BCG signals (B-HRV) of 18 healthy volunteers recorded by a force plate, and to evaluate the accuracy of estimating HRV indices, in both time and frequency domains, in comparison with the conventional ECG-derived method (E-HRV).

\section{Materials}

\subsection{Data acquisition}

BCG signals (sampling frequency of $960 \mathrm{~Hz}$ ) were acquired by a multicomponent biomechanical force plate (Type 9286B, Kistler $囚$ ), simultaneously to the ECG signal (sampling frequency of $2048 \mathrm{~Hz}$ ), in a group of 18 healthy volunteers (11 females). Study population characteristics are presented in Table 1.

Table 1. Study population characteristics (mean \pm std)

\begin{tabular}{|l|cl|}
\hline Age & $31 \pm 7$ & years \\
Weight & $62 \pm 14$ & $\mathrm{Kg}$ \\
Height & $1.67 \pm 0.11$ & $\mathrm{~m}$ \\
BMI & $22 \pm 3$ & $\mathrm{Kg} / \mathrm{m}^{2}$ \\
\hline
\end{tabular}

Two 5-minute recordings, in standing (ST) and supine (SUP) positions, were performed for each subject. During 
these periods subjects were asked to stay quiet, without voluntary movements, in order to minimize motion artifacts and ensuring that force and movement changes were mainly due to mass motion inside the body. Although the three orthogonal components of the reaction force were recorded, we focused only on the longitudinal head-to-foot component, as all the informative content was mainly projected into it.

\section{Methods}

\subsection{Preprocessing}

Pre-processing stage included automatic QRS detection and $\mathrm{T}$ wave delineation (Tonset, $\mathrm{T}_{\text {apex }}$ ) from the ECG signal using a wavelet-based ECG delineator [3], followed by the ECG-BCG synchronization. BCG signal was then filtered (band pass filtering $0.1-30 \mathrm{~Hz}$ ) in order to remove noise and other out-of-band components.

\subsection{Wave detection and series extraction}

A novel algorithm for the detection of systolic BCG waves $(\mathrm{H}, \mathrm{I}, \mathrm{J}, \mathrm{K})$ based on a max-min procedure was developed. In this first stage, it was implemented considering the information from the ECG signal (Rpeak, Tonset and Tapex marks) and defining physiological time intervals corresponding to the sequence of mechanical cardiac events within the whole beat. Defining the ECG and BCG signals as $x_{E C G}(n)$ and $x_{B C G}(n)$ respectively, four searching intervals were set, one for each wave:

1. J wave: it is the main positive wave occurring late in systole, corresponding to blood acceleration when ejected into the descending aorta. It was searched within a time interval defined around $\mathrm{T}$ wave occurrence:

$$
\begin{gathered}
n_{\mathrm{J}_{i}}=\arg \max _{n}\left\{x_{B C G}(n)\right\}, \\
n \in\left[n_{\mathrm{T}_{i}}-1 / 9 R R\left(n_{i+1}\right), n_{\mathrm{T}_{i}}+1 / 9 R R\left(n_{i+1}\right)\right]
\end{gathered}
$$

2. H wave: it represents the first maximum peak synchronous to the isovolumetric contraction. It was searched between the Rpeak and Tonset:

$$
\begin{gathered}
n_{\mathrm{H}_{i}}=\arg \max _{n}\left\{x_{B C G}(n)\right\} \\
n \in\left[n_{\mathrm{R}_{i}}+1 / 20 R R\left(n_{i+1}\right), n_{\mathrm{Ton}_{i}}-1 / 20 R R\left(n_{i+1}\right)\right](2)
\end{gathered}
$$

3. I wave: it is the first minimum wave at the onset of ejection (early systole), corresponding to blood acceleration in the ascending aorta and pulmonary arteries. It was defined as the minimum between $\mathrm{H}$ and $\mathrm{J}$ waves:

$$
n_{\mathrm{I}_{i}}=\underset{n}{\arg \min }\left\{x_{B C G}(n)\right\}, n \in\left[n_{\mathrm{H}_{i}}, n_{\mathrm{J}_{i}}\right]
$$

4. K wave: it is the second negative deflection associated to the descending slope of femoral pulse. It is synchronized with the second heart's sound, and so with semilunar valves' closure. It is searched in an interval starting at $\mathrm{J}$ wave

$$
\begin{aligned}
& n_{\mathrm{K}_{i}}=\arg \min _{n}\left\{x_{B C G}(n)\right\}, \\
& n \in\left[n_{\mathrm{J}_{i}}, n_{\mathrm{J}_{i}}+1 / 5 R R\left(n_{i+1}\right)\right]
\end{aligned}
$$

These searching conditions were valid for supine position, while in standing, $\mathrm{J}$ and $\mathrm{H}$ waves became negative and I and $\mathrm{K}$ waves positive, as BCG signal inverted its polarity.
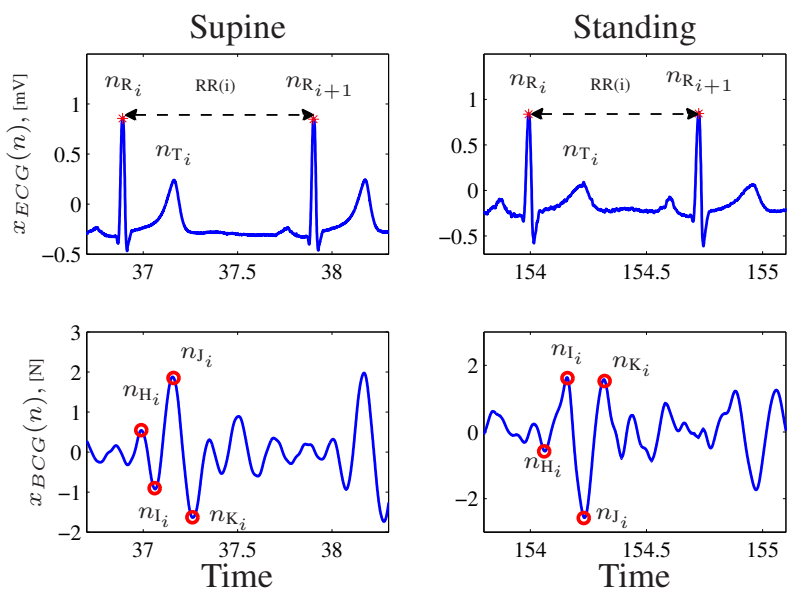

Figure 1. Examples of synchronized ECG and BCG signals in supine and standing positions, with overimposed the definition of the detected main waves.

After beat-to-beat fidutial point extraction, temporal BCG-series (HH, II, JJ and KK) were computed and validated for direct extraction of beat duration series against the conventional gold standard, the RR series, directly extracted from the ECG signal.

\subsection{HRV analysis}

Classical time and frequency-domain indices from both B-HRV and E-HRV were estimated for all 5-min recordings, where stationarity of the signal is assumed [1].

- Time domain: mean normal to normal interval (NN), standard deviation of NN intervals (SDNN) and root mean square of successive differences of adjacent NN intervals (RMSSD).

- Frequency domain: autoregressive (AR) spectral analysis was performed (Akaike criterion for choosing the model order) and spectral parameters computed, including normalized power in the low $(0.05-0.14 \mathrm{~Hz})$ and high $(\geq 0.15 \mathrm{~Hz})$ frequency bands $\left(\mathrm{P}_{\mathrm{LFn}}\right.$ and $\left.\mathrm{P}_{\mathrm{HFn}}\right)$, generally associated with sympathetic and parasympathetic responses, respectively. This analysis was conducted to test which, among all BCG-series, provides the most accurate power spectral density (PSD) compared to the PSD of the RR series, in both supine and standing positions. 
Table 2. Results of time and frequency HRV analysis in supine and standing positions expressed as median $\left(25^{\text {th }} ; 75^{\text {th }}\right.$ percentiles).*: $p \leq 0.05$ SUP vs ST.

\begin{tabular}{|c|c|c|c|c|c|c|c|c|c|c|}
\hline & \multicolumn{5}{|c|}{ Supine } & \multicolumn{5}{|c|}{ Standing } \\
\hline & $\begin{array}{l}\text { NN } \\
(\mathrm{ms})\end{array}$ & $\begin{array}{c}\text { SDNN } \\
(\mathrm{ms})\end{array}$ & $\begin{array}{c}\text { RMSSD } \\
(\mathrm{ms})\end{array}$ & $\begin{array}{l}\mathbf{P}_{\mathrm{LFn}} \\
\text { (n.u.) }\end{array}$ & $\begin{array}{l}\mathbf{P}_{\mathrm{HFn}} \\
\text { (n.u.) }\end{array}$ & $\begin{array}{l}\mathbf{N N} \\
(\mathrm{ms})\end{array}$ & $\begin{array}{c}\text { SDNN } \\
(\mathrm{ms})\end{array}$ & $\begin{array}{c}\text { RMSSD } \\
(\mathrm{ms})\end{array}$ & $\begin{array}{l}\mathbf{P}_{\text {LFn }} \\
\text { (n.u.) }\end{array}$ & $\begin{array}{l}\mathbf{P}_{\mathrm{HFn}} \\
\text { (n.u.) }\end{array}$ \\
\hline \multirow[t]{2}{*}{$\mathbf{R R}$} & 952 & 56 & 45 & 0.52 & 0.48 & $667 *$ & $40^{*}$ & $18^{*}$ & $0.88^{*}$ & $0.12 *$ \\
\hline & (792-989) & $(37-72)$ & $(26-63)$ & $(0.30-0.79)$ & $(0.20-0.69)$ & $(627-734)$ & $(31-54)$ & (14-24) & $(0.83-0.95)$ & $(0.07-0.17)$ \\
\hline \multirow[t]{2}{*}{ HH } & 951 & 60 & 58 & 0.37 & 0.63 & $667 *$ & 55 & 62 & 0.35 & 0.65 \\
\hline & (791-989) & $(41-73)$ & $(35-66)$ & $(0.29-0.51)$ & $(0.48-0.68)$ & $(627-734)$ & $(45-62)$ & $(55-65)$ & $(0.23-0.43)$ & $(0.57-0.76)$ \\
\hline \multirow[t]{2}{*}{ II } & 952 & 57 & 48 & 0.48 & 0.52 & 668* & 49 & 42 & 0.44 & 0.56 \\
\hline & $(792-989)$ & $(39-72)$ & $(27-63)$ & $(0.30-0.62)$ & $(0.37-0.70)$ & $(627-734)$ & $(45-58)$ & $(36-63)$ & $(0.30-0.66)$ & $(0.30-0.62)$ \\
\hline \multirow[t]{2}{*}{ JJ } & 952 & 57 & 48 & 0.48 & 0.52 & $668 *$ & 46 & 37 & 0.52 & 0.48 \\
\hline & (792-989) & $(38-72)$ & $(28-63)$ & $(0.31-0.66)$ & $(0.34-0.69)$ & $(627-734)$ & $(38-59)$ & $(34-46)$ & $(0.47-0.63)$ & $(0.37-0.53)$ \\
\hline \multirow[t]{2}{*}{ KK } & 951 & 58 & 48 & 0.47 & 0.53 & $668^{*}$ & 50 & 43 & 0.40 & 0.59 \\
\hline & (791-988) & $(39-73)$ & $(33-63)$ & $(0.32-0.67)$ & $(0.33-0.68)$ & $(627-734)$ & $(38-61)$ & $(34-56)$ & $(0.35-0.61)$ & $(0.39-0.65)$ \\
\hline
\end{tabular}

\subsection{Statistical analysis}

Due to non-Gaussian distribution, data were presented as median and 25th and 75th percentiles acorss subjects, unless otherwise specified. Linear correlation and BlandAltman analyses were applied to beat-to-beat measurements, to define which, among all BCG series, is the best surrogate in respect to the RR series computed from the ECG. To evaluate significance of changes induced by posture, a non-parametric Wilcoxon rank test was applied between SUP and ST values. Null hypothesis was rejected when $\mathrm{p} \leq 0.05$.

\section{Results}

Table 2 shows the results of classical time and frequency indices derived from E-HRV and B-HRV for each posture.

Comparison of BCG temporal series with the RR series were more accurate in SUP than in ST, with higher $\mathrm{R}^{2}$ values and narrower confidence $(\mathrm{CI}, \pm 2$ std) intervals (see Table 3). For both postures, the JJ series was the best correlated and most accurate measure of the heart cycle duration in respect to the RR .

Table 3. Correlation and Bland-Altman results for the comparison of BCG series with the RR series.**: $p \leq$ 0.01 .

\begin{tabular}{c|ccc|ccc|}
\cline { 2 - 7 } & \multicolumn{3}{|c|}{ Supine } & \multicolumn{3}{c|}{ Standing } \\
\cline { 2 - 7 } & $\mathbf{R}^{2} * *$ & $\begin{array}{c}\text { Bias } \\
(\mathrm{ms})\end{array}$ & $\begin{array}{c}\text { CI } \\
(\mathrm{ms})\end{array}$ & $\mathbf{R}^{2} * *$ & $\begin{array}{c}\text { Bias } \\
(\mathrm{ms})\end{array}$ & $\begin{array}{c}\text { CI } \\
(\mathrm{ms})\end{array}$ \\
\hline $\mathbf{H H}$ & 0.990 & 0.02 & \pm 35 & 0.908 & 0.01 & \pm 74 \\
$\mathbf{I I}$ & 0.997 & 0.01 & \pm 19 & 0.947 & 0.04 & \pm 55 \\
$\mathbf{J J}$ & 0.998 & 0.01 & \pm 17 & 0.968 & -0.01 & \pm 42 \\
$\mathbf{K K}$ & 0.995 & 0.03 & \pm 24 & 0.950 & 0.07 & \pm 53 \\
\hline
\end{tabular}

Considering this observation, only results obtained from spectral analysis of the JJ series were evaluated. Figure 2 shows an example of the estimated PSD of the RR and the JJ series from one subject in both SUP and ST positions. When comparing RR and JJ spectral indices (see Table 4), only for SUP both $\mathrm{P}_{\mathrm{LFn}}$ and $\mathrm{P}_{\mathrm{HFn}}$ presented a high and significant correlation. From Bland-Altman, $\mathrm{P}_{\mathrm{LFn}}$ from JJ represented an acceptable surrogate $(\mathrm{CI}= \pm 30 \%)$, while $\mathrm{P}_{\mathrm{HFn}}$ showed larger limits of agreement $(\mathrm{CI}= \pm 71 \%)$.
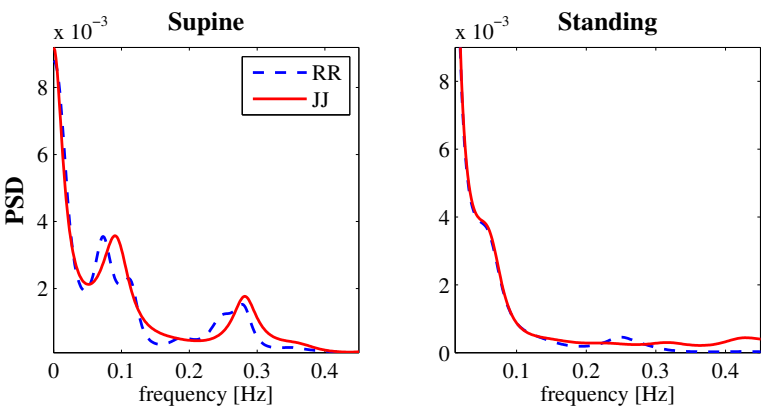

Figure 2. Example of PSD of the RR and the JJ series extracted from one subject in both SUP and ST positions.

Table 4. Correlation and Bland-altman results for the comparison of spectral B-HRV and E-HRV parameters. **: $p \leq 0.01$.

\begin{tabular}{c|ccc|ccc|}
\cline { 2 - 7 } & \multicolumn{3}{c|}{ Supine } & \multicolumn{3}{c|}{ Standing } \\
\cline { 2 - 7 } & $\mathbf{R}^{2} * *$ & $\begin{array}{c}\text { Bias } \\
(\%)\end{array}$ & $\begin{array}{c}\text { CI } \\
(\%)\end{array}$ & $\mathbf{R}^{2}$ & $\begin{array}{c}\text { Bias } \\
\text { (n.u.) }\end{array}$ & $\begin{array}{c}\text { CI } \\
\text { (n.u.) }\end{array}$ \\
\hline $\mathbf{P}_{\mathrm{LFn}}$ & 0.916 & -5 & $\pm 30 \%$ & 0.084 & 0.32 & \pm 0.33 \\
$\mathbf{P}_{\mathrm{HFn}}$ & 0.888 & +21 & $\pm 71 \%$ & 0.068 & -0.31 & \pm 0.34 \\
\hline
\end{tabular}




\subsection{Physiological analysis results}

From SUP to ST, there was a significant decrease in both the duration and the total variance of all intervals, as expected from physiology. From the analysis of the RR series in frequency domain, a significant increase in the $\mathrm{P}_{\mathrm{LFn}}$ together with a decrease in the $\mathrm{P}_{\mathrm{HFn}}$ was observed passing from SUP to ST (see table 2). As regards the BCG series, only the JJ series presented a similar trend in both parameters, though with not significant changes.

\section{Discussion and conclusions}

The purpose of the study was to evaluate the potential usefulness of BCG signal for non-invasive monitoring of heart beat duration and HRV, in comparison to high-fidelity ECG acquisition and RR series extraction.

Our results confirmed the feasibility of extracting heart rate information from all the BCG series $(\mathrm{HH}, \mathrm{II}, \mathrm{JJ}$ and $\mathrm{KK}$ ) by force platform, with higher accuracy in supine position, supporting the usefulness of B-HRV indices in the time domain. In particular, the JJ series represented the best surrogate of the RR measurement. Compared to previous studies, average estimation errors were found reduced compared to [4] in supine position, while in standing, compared to [5], a reduced bias but wider CI was obtained. This discrepancy could probably be due to the fact that in [5] analysis was performed in shorter time windows (10 $\mathrm{sec})$.

As regards the evaluation of the symphato-vagal balance in terms of frequency components, the $\mathrm{JJ}$ series provided only a good estimate of $\mathrm{P}_{\mathrm{LFn}}$ limited to the supine position. This difficulty in capturing the HF oscillations using JJ series could be due to the fact that $\mathrm{J}$ wave from the BCG is smoother than the Rpeak in the ECG, thus limiting accurate detection by introducing noise. Also involuntary body movements could have interfered on the BCG.

Easily overcoming the actual limitation of being the algorithm ECG-dependent for extracting BCG waves, and considering this as an exploratory study, advances in signal processing techniques and the possibility of embedding BCG sensors in objects of common use (such us scales, seats, beds and even smartphones) offer the opportunity of extracting heart rate information in a simple and noninvasive way, thus satisfying new demands and trends of home e-health systems for daily cardiac monitoring.

\section{Acknowledgements}

This research is supported by the Belgian Federal Science Policy Office (BELSPO) via the ESA PRODEX program. E.G. Caiani is supported by the Italian Space Agency (contract 2013-064-R.0).

\section{References}

[1] The Task Force of ESC and NASPE. Heart rate variability: Standards of measurement, physiological interpretation, and clinical use. Eur Heart J 1996;93:1043-1065

[2] Gubner RS, Rodstein M, Ungerleider HE. Ballistocardiography - appraisal of technic physiologic principles and clinical value. Circulation 1953;7:268-286

[3] Martínez JP, Almeida R, Olmos S, Rocha AP, Laguna P. Wavelet-based ECG delineator: evaluation on standard databases. IEEE Trans Biomed Eng 2004;51:570-581

[4] Bruser C, Stadlthanner K, de Waele S, Leonhardt S. Adaptive Beat-to-Beat Heart Rate Estimation in Ballistocardiograms. IEEE Trans Inf Technol Biomed 2011;15(5):778786

[5] R González-Landaeta R, Casas O, Pallás-Areny, R. Heart rate detection from an electronic weighing scale. Physiol. Meas. 2008;29(8):979-988

Address for correspondence:

Alba Martín

DEIB, Politecnico di Milano Piazza Leonardo da Vinci, 32, 20.133, Milano, Italy

albapilar.martin@polimi.it 